\title{
TIME DEPENDENT BLR PHOTOIONIZATION MODELS
}

\author{
P.T. O'BRIEN and M.R. GOAD \\ Department of Physics \& Astronomy, University College London
}

\begin{abstract}
Recent intensive campaigns to monitor the variable broad emission lines of AGN have provided a wealth of observational data. To reliably derive the BLR structure from such data requires a detailed comparison with physically realistic models. To provide such models we have combined photoionization calculations with a BLR modelling code. We find that the line emission is significantly anisotropic for the majority of lines at some radii in our models, particularly where the ionization parameter is large. As both anisotropic line emission and negative responsivity can have a substantial impact on the form of response functions, they must be taken into account when comparing BLR models to monitoring data. Differentiating between the effects of anisotropy and negative responsivity requires comparison of the variability behaviour of lines covering a wide range in ionization state with detailed photoionization models similar to those presented here. Failure to take such physical effects into account will lead to incorrect conclusions regarding the BLR structure.

Based on the form of the anisotropic response functions for our models, we argue that in NGC5548 the high ionization lines (including CIII] $\lambda 1909$ ) can be explained by a spherical BLR component which has a significant radial ionization parameter gradient. The clouds in this component may be optically thin at the Lyman limit in the inner part of the BLR due to the high ionization parameter. The bulk of the low ionization lines, and in particular almost all of the Balmer line emission, arises from a non-spherical BLR component, possibly an accretion disc.

We have also investigated the accuracy of the linear response approximation by implicitly calculating the emissivity as a function of continuum level. For several lines the response is significantly non-linear over the typical continuum variability range of AGN. The form of the response functions is therefore luminosity dependent. Work is under way to incorporate this effect into the maximum-entropy-method used to recover response functions from monitoring data.
\end{abstract}

Key words: AGN, Photoionization Models, Emission Lines

T. J.-L. Courvoisier and A. Blecha: Multi-Wavelength Continuum Emission of AGN, 453.

(C) 1994 IAU. Printed in the Netherlands. 